\title{
Hydrogen Bonding of Water Confined in Controlled-Pore Glass 10-75 Studied by ${ }^{1} \mathbf{H}-$ Solid State NMR
}

\author{
By A. Vyalikh ${ }^{1}$, Th. Emmler ${ }^{1}$, B. Grünberg ${ }^{1}, \mathrm{Y}_{\mathrm{X}} \mathrm{Xu}^{1}$, I. Shenderovich ${ }^{1}$, \\ G. H. Findenegg ${ }^{2}$, H.-H. Limbach ${ }^{1}$, and G. Buntkowsky ${ }^{3, *}$ \\ ${ }^{1}$ Freie Universität Berlin, Institut für Chemie, Takustraße 3, D-14195 Berlin, Germany \\ 2 Technische Universität Berlin, Stranski-Laboratorium für Physikalische und \\ Theoretische Chemie, Straße des 17. Juni 112, D-10623 Berlin, Germany \\ ${ }^{3}$ FSU Jena, Institut für Physikalische Chemie, Helmholtzweg 4, D-07743 Jena, \\ Germany
}

(Received May 1, 2006; accepted in revised form August 7, 2006)

\section{Hydrogen Bonding / Water / Controlled Pore Glass / ${ }^{1}$ H-NMR Spectroscopy / Pore Condensation}

\begin{abstract}
The adsorption of water in the mesoporous silica material with cylindrical pores of uniform diameter, Controlled Pore Glass 10-75 (CPG), was studied by ${ }^{1} \mathrm{H}-\mathrm{MAS}$ solid state NMR spectroscopy. From the NMR spectra it is evident that inside the mesopores of the silica different water environments exist, which are characterized by their individual chemical shift. All observed hydrogen atoms are either surface $-\mathrm{SiOH}$ groups or hydrogen bonded water molecules. It is found that there exist some stronger bound water molecules on the surface which are not removable even by heating at a vacuum pump. As a tentative assignment these water molecules are attributed to surface defects or inaccessible cavities in the CPG 10-75. At intermediate water filling levels, the principal signal is a single NMR line with continuously varying chemical shift. This finding is interpreted as the result of a radial water filling mechanism. That is, the filling of the pore grows from the pore surface towards the pore axis. Finally it is shown that water is a sensor for surface and structural inhomogeneity and that a coexistence of inner pore and outer bulk water exists in the system.
\end{abstract}

\section{Introduction}

Silica materials with large surfaces are important technical materials for filtering processes in food technology. In particular they are employed to remove unwanted proteins from aqueous-protein solutions while leaving the desired proteins. Until now, the application of these silica materials is based on a purely

\footnotetext{
* Corresponding author. E-mail: gerd.buntkowsky@uni-jena.de
}

Available under

only the rights of use according to UrhG 
empirical basis. Understanding the interactions of the protein with the silica surface could greatly help in designing more efficient surface morphologies. Since water is the solvent which mediates the interaction of the protein with the surface, the first steps in the characterization of the surface protein interactions are studies of the interaction of water with both silica and proteins. In a previous work we therefore characterized water in well defined silica materials which allows a deep understanding of the interactions on the molecular level $[1,2]$. In the present work we compare these results to the behavior of water in a less ordered and thus less well defined porous silica glass, which is expected to be more inhomogenous and thus more close to technical materials. With these experiments we want to see, to which extend the results from the ordered materials can be transferred to the technical materials.

Water is the primary solvent for most naturally occurring chemical and biological reactions. The special physical and solvent properties of the water stem largely from its extraordinary internal cohesiveness, compared to most other liquids of similar molecular weights. This cohesiveness is mainly the result of the water molecules high polarity and their ability to form hydrogen bonded networks among themselves, as for example in the frozen phase or in the bulk liquid phase. In restricted geometries the water molecules can also interact with the surfaces through hydrophobic and hydrophilic interactions and hydrogen bond interactions; hence there is a competition between the surface-liquid and liquid-liquid interactions. This competition can lead to interesting new structures of the water, which are not observed in bulk water. In particular often at least partial ordering of the water molecules in the vicinity of the confining surface is found. Important examples of such systems are water molecules enclosed in porous media like zeolites [3] or cements [4], or water molecules in hydration shells of proteins [5-11]. Specifically, the existence of two kinds of water in pores, free water in the center of the pore, and bound water near the pore surface, has been established by a variety of experimental techniques [3,12-22]. Recently such surface and core water phases were observed by ${ }^{1} \mathrm{H}$-MAS solid state NMR spectroscopy in mesoporous silica materials MCM-41 [23] and SBA-15 [24] with parallel arrangement of cylindrical pores [1]. There a dependence of the pore filling mechanism on the pore diameter was found. Besides the interesting application potential for technical processes these materials are also powerful NMR models for the study of surface-fluid interactions [1, 2, 25-29].

In the present work we study water as a guest molecule in the mesopores of a Controlled Pore Glass (CPG). This material constitutes an amorphous network of pores of uniform size in a range between 4 and $10^{4} \mathrm{~nm}$ [30]. Due to their wide range of available pore sizes they are very versatile molecular sieves. The pore cross sections of the Controlled Pore Glass are approximately circular and therefore are thought of as having roughly cylindrical pores. They are prepared by a spinodal decomposition process that involves quenching a liquid 
mixture of oxides into the two-liquid-phase region. The pore size is determined by the time at which the quenched mixture is kept at an intermediate temperature, before the final quench to room temperature. Such glasses have a narrow distribution of pore diameters, and have been widely used in experimental studies. The interpretation of such experimental results has often relied on models in which the pore structure is presented by a set of straight, cylindrical, nonconnected pores having a range of diameters. However, electron micrographs of these glasses [31] show a sponge-like networked structure with winding pores, and simple straight cylinder models of these materials do not capture some important confinement effects.

Analyzing water structures inside the porous space can provide a detailed understanding the water-surface interaction on the molecular level and this knowledge may help later in studying the interactions of these surfaces with biological molecules. For this analysis a molecular observable must be studied, which is sensitive to the structure and binding of individual water molecules. In the present work the ${ }^{1} \mathrm{H}$-chemical shift of the water molecules is employed as such an observable. Its value depends strongly on the structural and dynamical properties of the hydrogen atoms. Since the observed phases are often ordered, i.e. anisotropic, one can expect at least some residual anisotropy in the NMR parameters that contributes to the line-width of the observed spectrum.

The line-width of an NMR signal depends strongly on the microscopic environment of the nucleus under study. Interactions such as the chemical shift and dipole-dipole coupling between neighboring spins are anisotropic and impose a dependence on the NMR frequency based on the orientation of the spin or molecule with respect to the main magnetic field direction.

In liquid samples the fast isotropic motion of the molecules averages the anisotropic interactions, resulting in an isotropic chemical shift frequency and removal of the line broadening due to dipolar coupling. In solid samples on the other hand, the lack of molecular mobility results in broad lines. These interactions can be averaged out by spinning the sample at the magic angle with the direction of the external magnetic field (MAS-NMR). Therefore the heterogeneous system of water-Controlled Pore Glass is studied by ${ }^{1} \mathrm{H}-\mathrm{MAS}-\mathrm{NMR}$ spectroscopy.

\section{Experimental section}

\subsection{Sample preparation}

The Controlled Pore Glass (CPG 10-75) material supplied by Fluka consists of an interconnected pore network with a narrow pore size distribution (average $\Delta r<0.5 \mathrm{~nm}$ ) and an average pore diameter of $10.3 \mathrm{~nm}$. The specific surface area (after Brunauer, Emmett, Teller characterization, BET) of CPG 10-75 is $182 \mathrm{~m}^{2} / \mathrm{g}$ and the specific pore volume is $0.47 \mathrm{~cm}^{3} / \mathrm{g}$. Any organic impurities 
and physisorbed water in the samples were removed by storing them in concentrated $\mathrm{H}_{2} \mathrm{SO}_{4}$ for three days, followed by Soxhlet extraction with de-ionized water for two days and heating at $150^{\circ} \mathrm{C}$ at $10^{-6}$ mbar for $24 \mathrm{~h}$. CPG $10-75$ was loaded into a $4 \mathrm{~mm}$ o. d. NMR rotor to a height of ca. $1 \mathrm{~cm}$. The sample was heated under vacuum during $24 \mathrm{~h}$ using a vacuum line to remove any moisture from the tube walls and the silica, then filled with argon and weighed on an analytical balance (METTLER) with an accuracy of $\pm 0.05 \mathrm{mg}$. The obtained weight $(35.55 \pm 0.05 \mathrm{mg})$ and the specific characteristic of the pore volume were used for the determination of the total pore volume in the sample. Then the defined amount of normal water $\left(\mathrm{H}_{2} \mathrm{O}\right)$ was added to the sample employing a syringe, the sample was weighed and left for a few hours to achieve saturation of the pores by water. The removal of water was realized by two different ways depending on water amount in the silica. At high water contents the sample was simply heated with a heat gun for a few minutes for water evaporation. At low water contents the sample was placed into a vacuum line to actively pump out the water confined in the silica pores or adsorbed on the pore walls. The amount of removed water was controlled by temperature and time of the pumping process. After this procedure the ${ }^{1} \mathrm{H}-\mathrm{MAS}-\mathrm{NMR}$ spectrum as a function of the pore filling was measured. The sample was weighed before and after each experiment to ensure that no water losses took place during the measurements. For the ${ }^{1} \mathrm{H}-\mathrm{NMR}$ experiment the rotor was closed with a rotor cap, which seals the rotor sufficiently tight to prevent the evaporation of the water during the ${ }^{1} \mathrm{H}-\mathrm{NMR}$ measurements. Then the controlled amount of water was removed, the sample weighed and the ${ }^{1} \mathrm{H}-\mathrm{NMR}$ spectrum was recorded. This procedure was repeated until a sole line at $1.74 \mathrm{ppm}$ appeared in the spectrum and its intensity has not decreased, which is characteristic for surface silanol groups. Precise data of the samples are given in Table 1.

\section{$2.2{ }^{1}$ H-solid-state-NMR-spectroscopy}

All ${ }^{1} \mathrm{H}-\mathrm{NMR}$ measurements were performed on a solid state NMR spectrometer, operating at 7 Tesla, equipped with a Bruker $4 \mathrm{~mm}$ single resonance ${ }^{1} \mathrm{H}$-CRAMPS probe. The room temperature magic angle spinning (MAS, $10000 \mathrm{~Hz}$ ) NMR experiments were performed employing a one- $90^{\circ}$-pulse sequence with a full CYCLOPS phase cycling. $90^{\circ}$-pulse length was $3.9 \mu$ s and the sweep width was $200 \mathrm{kHz}$. All ${ }^{1} \mathrm{H}-\mathrm{NMR}$ chemical shift values are referenced to the signal from TMS. Before the ${ }^{1} \mathrm{H}-\mathrm{NMR}$ experiment of each sample TMS was measured as an external standard. The recycling delay time was determined by the water longitudinal relaxation time. Because the relaxation time depends strongly on water content in the pores, the recycle delay time decreased from $10 \mathrm{~s}$ in saturated samples to $1 \mathrm{~s}$ in the unsaturated samples. 
Table 1. Water content in the CPG 10-75: The first column shows the identifier for each sample (see the spectra in Fig. 1), $n\left(\mathrm{H}_{2} \mathrm{O}\right)$ and $V$ are the estimated number of $\mathrm{H}_{2} \mathrm{O}$ molecules on the surface per $\mathrm{nm}^{2}$ and the content of $\mathrm{H}_{2} \mathrm{O}$ in pore volume (calculated with respect to the specific surface area $S_{\mathrm{p}}$ and the specific pore volume $V_{\mathrm{p}}$, correspondingly). The last column shows the mass of water in each sample. The percentage values give the nominal filling of the sample. In the experiments it is shown that part of the pore volume is inaccessible to water.

\begin{tabular}{cccc}
\hline sample & $n\left(\mathrm{H}_{2} \mathrm{O}\right)$, pro $\mathrm{nm}^{2}$ & $V, \%$ & $m, \mathrm{mg}$ \\
\hline $\mathbf{a}$ & 104.5 & 134.0 & 20.0 \\
$\mathbf{b}$ & 67.3 & 86.3 & 12.9 \\
$\mathbf{c}$ & 16.4 & 20.9 & 3.1 \\
$\mathbf{d}$ & 13.7 & 17.6 & 2.6 \\
$\mathbf{e}$ & 2.5 & 3.2 & 0.5 \\
$\mathbf{f}$ & 0.8 & 1.0 & 0.1 \\
$\mathbf{g}$ & $<0.05$ & $<0.05$ & 0.0 \\
\hline
\end{tabular}

\section{Results and discussions}

\subsection{Experimental results}

Figure 1 displays the ${ }^{1} \mathrm{H}-\mathrm{MAS}-\mathrm{NMR}$ spectra of water in CPG 10-75 for different water contents recorded at a MAS frequency of $10 \mathrm{kHz}$. All presented spectra are normalized to their maximal intensity. The spectra, in general, exhibit several resolved lines with typical line-widths of $(0.2-0.7) \mathrm{ppm}$. The ${ }^{1} \mathrm{H}-\mathrm{MAS}-\mathrm{NMR}$ spectrum which was measured after drying on the vacuum line (g) exhibits a single line at $\delta=1.74 \mathrm{ppm}$. This single line is labeled as $\mathbf{I}$. However, even in the nominally completely dried sample some spectral intensity between two and three ppm is observed. To remove the remaining water the sample was additionally dried for $48 \mathrm{~h}$ at $100{ }^{\circ} \mathrm{C}$ on the vacuum line. The ${ }^{1} \mathrm{H}-\mathrm{MAS}-\mathrm{NMR}$ spectrum (not shown here) measured after that did not reveal a decrease of spectral intensity. This is evidence for residual water molecules, which are probably stuck in the pore defects and hydrogen bonded to silica surface. Figure 1 (f) displays the spectrum with the lowest water content of $1.0 \%$ nominal. Here the spectrum is still dominated by $\mathbf{I}$, but an increase of the line-width and a slight low field shift to $1.78 \mathrm{ppm}$ are observed. Additionally a low field shoulder of the line with a broad distribution of spectral frequencies from two to four ppm (II) and a high field peak of the small intensity at $0.88 \mathrm{ppm}$ (III) are visible. Upon further increase of the water content to $3.2 \%$ nominal (e) the broadening of the $1.78 \mathrm{ppm}$ line continues and a slight shift of the peak (III) to $0.91 \mathrm{ppm}$ is observed. The lower field shoulder II grows up to a single line at $3.0 \mathrm{ppm}$ that finally starts to dominate the spectrum (d) in the sample with $17.6 \%$ nominal water. Upon further increase of the water content to $20.0 \%$ nominal (c) the line (II) is broadened and low field shifted to $3.2 \mathrm{ppm}$. The spectrum is accompanied by two peaks with small intensity at $1.26 \mathrm{ppm}$ 


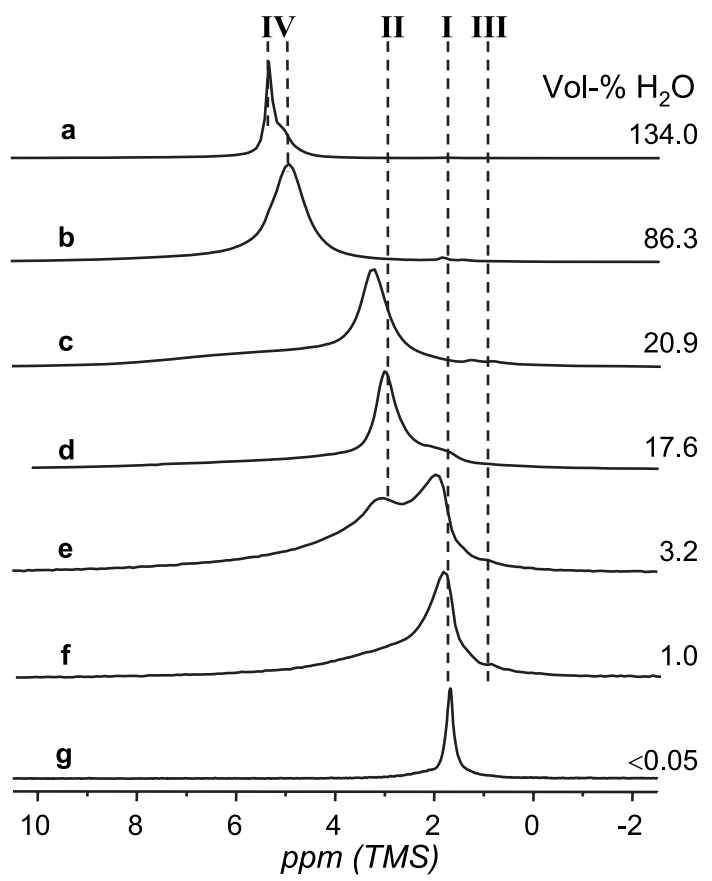

Fig. 1. Experimental ${ }^{1} \mathrm{H}-\mathrm{MAS}$ solid state NMR spectra $(10 \mathrm{kHz})$ of water in CPG 10-75 at variable water content. The letters refer to Table 1.

and $0.80 \mathrm{ppm}$. In the spectrum with $86.3 \%$ nominal water filling (b) a new signal appears at $4.9 \mathrm{ppm}(\mathbf{I V})$, while two high field peaks with small intensity (III) are shifted to $1.79 \mathrm{ppm}$ and $1.39 \mathrm{ppm}$. The spectrum (a) from an overloaded sample with $134 \%$ nominal water content represents a superposition of two lines: The signal IVa shifted to $5.0 \mathrm{ppm}$ and the signal at $5.3 \mathrm{ppm}$ (IVb) with dominating intensity. In addition to these relatively narrow lines a broad background signal, which covers the whole range from $1 \mathrm{ppm}$ to $8 \mathrm{ppm}$ is visible in the spectra (b)-(e) exhibiting a maximal intensity in the spectrum (c).

\subsection{Discussion}

From the NMR spectra shown above it is evident that inside the silica pores different water environments exist. They can be characterized by their apparent chemical shift, which is caused by the interplay of the individual chemical shift values of the different protons and the chemical exchange of the protons. In the following the experimental results are discussed with respect to the water structures inside the pore space. Figure 2 displays the different possible scenarios of water molecules hydrogen bonded to the silica surface or among each other or free. Each scenario is characterized by an individual ${ }^{1} \mathrm{H}$-chemical shift. While 
a)

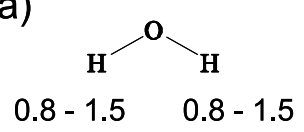

d)

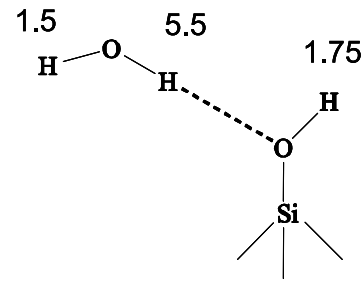

b)

$\left(\mathrm{H}_{2} \mathrm{O}\right) \mathbf{n}$

5.5

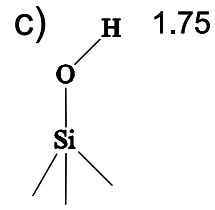

f) e)

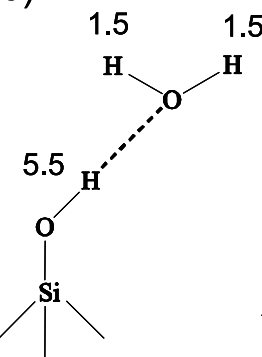

1.5

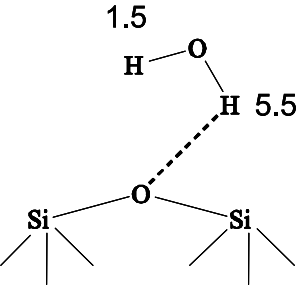

Fig. 2. Overview of possible -OH groups in the water/silica samples and the corresponding chemical shifts in ppm (TMS). Upper row: chemical shifts of the constituents of monomeric water, water clusters and silanol groups. Lower row: chemical shifts (adapted from Refs. $[1,32,35])$ observed in various hydrogen bonding scenarios.

in principle these ${ }^{1} \mathrm{H}$-chemical shifts are unique for a defined structure of the water interacting with the surface and other water molecules, in practice it must be taken into account that dynamic exchange effects like molecular reorientations of the water molecules, rotations of the surface $-\mathrm{SiOH}$ groups and proton transfer can and in general will cause changes of these chemical shifts that lead to complete or full averaging of the line positions. Nevertheless it is still possible to distinguish between different environments and thus determine the relative amounts of the individual species by virtue of the ${ }^{1} \mathrm{H}$-chemical shift, as discussed by Grünberg et al. [1].

Employing this chemical shift information the results of water in CPG 10-75 are discussed. In the dried sample (Fig. 1g) only a single resonance at $\delta=1.74 \mathrm{ppm}$ is observed. Comparing this value to the chemical shift of $-\mathrm{SiOH}$ protons in MCM-41 $(\delta=1.75 \mathrm{ppm})$ [32], it is evident that this signal can be attributed to free surface $-\mathrm{SiOH}$ protons. The presence of the background line at around $2 \mathrm{ppm}$ in nominally dry sample shows that not all water molecules are removable from CPG 10-75. That means that there is strongly bound water in the pores, for example due to structural inhomogeneities or structural defects, respectively water molecules confined in inaccessible places.

This finding can be compared with the mesoporous silica materials SBA-15 and MCM-41 studied by Grünberg et al. [1]. For comparison the corresponding spectra are reproduced below (Fig. 3). There it has been shown that in well ordered MCM-41 with a pore diameter of $3.3 \mathrm{~nm}$ all water molecules can be easily removed on a vacuum line by moderately heating the sample with 

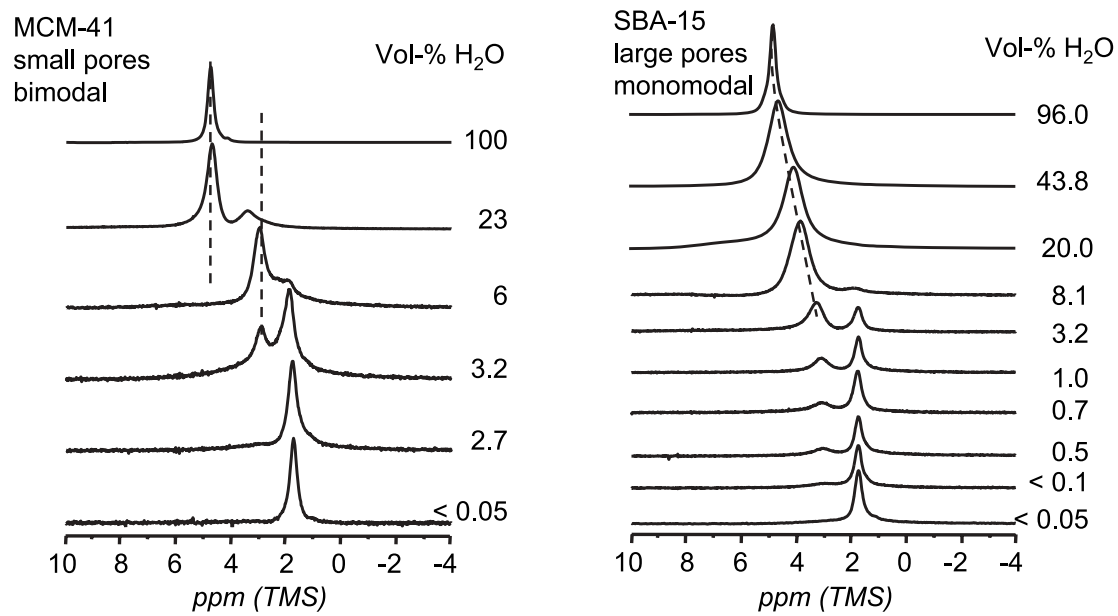

Fig. 3. Experimental ${ }^{1} \mathrm{H}-\mathrm{MAS}$ solid state NMR spectra $(10 \mathrm{kHz})$ of water in MCM-41 and SBA-15 (adapted from Ref. [1]).

a heat gun. This is obvious from the ${ }^{1} \mathrm{H}-\mathrm{NMR}$ spectrum exhibiting a single line at $1.74 \mathrm{ppm}$. In contrast to MCM-41, in SBA-15 sample with a pore diameter of $8.0 \mathrm{~nm}$ such a procedure did not cause a complete removal of all water from the pores, while only the drying on the vacuum line at $120^{\circ} \mathrm{C}$ during $24 \mathrm{~h}$ removed the remaining water from the sample. Thus the SBA-15 sample exhibits some kind of structural inhomogeneities which cause a stronger binding or trapping of water molecules. This observation is corroborated by the investigation of the surface roughness of SBA-15 and MCM-41 carried out by Shenderovich et al. [33]. In this work a fast rotational diffusion, combined with a surface hopping of the ${ }^{15} \mathrm{~N}$ and para- ${ }^{2} \mathrm{H}$ labeled pyridine molecules on the surfaces of the pores was observed. These combined motions cause a motional averaging of the ${ }^{15} \mathrm{~N}$ chemical shift anisotropy and ${ }^{2} \mathrm{H}$ quadrupolar tensors of the molecule. While in the case of SBA-15 a complete averaging to an isotropic line was observed, residual anisotropy has remained in MCM-41. The full averaging observed in SBA-15 is only possible in case of a surface with considerable roughness, owing to the presence of structural defects.

Since the general chemical and crystalline structures of MCM- 41 and SBA-15 are very similar with respect to the hydrogen bonding of the water molecules to the surface, these structural defects are the main difference between MCM-41 and SBA-15. Thus one can conclude that they are responsible for the trapping of the strongly bound water molecules on the surfaces.

In the case of the CPG 10-75 the remaining water is observed in the nominally dried sample, which leads to conclusion about considerable surface roughness of the CPG material. In comparison to SBA-15 even a longer ther- 
mal treatment of the CPG under vacuum does not provide an absolute removal of water from the sample. That means that there is not only the structural surface inhomogeneity, but also trapping sites that keep the water molecules. The most probable sources for such traps are microdefects with diameters much smaller than the average pore diameter. Therefore the confined molecules can not be easily removed from CPG 10-75.

Comparing the observed chemical shifts of the water signals at low filling levels $(2.0 \mathrm{ppm}-3.2 \mathrm{ppm})$ with the values given for the possible chemical shifts of $-\mathrm{OH}$ groups (see Fig. 2), it is evident that none of these shifts matches the observed shift. From this it follows that the observed shift is the result of the weighted averaging between different water species, caused by fast chemical exchange among them and consequently, all water molecules contribute to hydrogen bonds.

The average distance between surface - $\mathrm{OH}$ groups can be estimated from their surface densities. The number of $-\mathrm{OH}$ groups per surface unit for the CPG material to the best of our knowledge is not known. This value is supposed to depend strongly on the manufacturing process of the sample and to vary with calcination temperature and other conditions. But knowing the surface densities $[1,33], n_{\mathrm{OH}} \approx 3 \mathrm{~nm}^{-2}$ for MCM-41 and $n_{\mathrm{OH}} \approx 3.7 \mathrm{~nm}^{-2}$ for SBA-15 the averaged distance between surface -OH groups can be estimated as $0.58 \mathrm{~nm}$ and $0.52 \mathrm{~nm}$, respectively. These average distances are too large to be bridged by a single, hydrogen bonded water molecule. In other words only one of the two water protons can be in a hydrogen bond with surface $-\mathrm{OH}$ groups in a given moment. Since both water protons are found to be magnetically equivalent it follows that they are in fast exchange. The assumption of (i) fast exchange of only the two water protons and (ii) a fixed - $\mathrm{SiOH}$ $\ldots \mathrm{OH}_{2}$ hydrogen bond would give a line of the water protons at $(1.5 \mathrm{ppm}+$ $5.5 \mathrm{ppm}) / 2=3.5 \mathrm{ppm}$. Since the observed line of the water proton is below $3 \mathrm{ppm}$ for low filling factors one can conclude that a more complicated exchange process, which involves the proton of the $-\mathrm{SiOH}$ group occurs. The simplest mechanism is the mutual exchange of the two water protons with the $-\mathrm{SiOH}$ proton.

Assuming a symmetric exchange between all three positions, such an exchange process would cause an average line at $(1.5 \mathrm{ppm}+1.75 \mathrm{ppm}+$ $5.5 \mathrm{ppm}) / 3=2.9 \mathrm{ppm}$ for configuration (d) (Fig. 2$)$ and $(1.5 \mathrm{ppm}+1.5 \mathrm{ppm}$ $+5.5 \mathrm{ppm}) / 3=2.8 \mathrm{ppm}$ for configuration (e) (Fig. 2), which are closer to the experimentally observed chemical shifts. In practice one can assume that this process is not a simple chemical exchange of the three protons but a surface hopping of the water molecule, which is accompanied by the formation and breaking of covalent $-\mathrm{OH}$ bonds and $\mathrm{O} \ldots \mathrm{H}$ hydrogen bonds.

Upon further increase of the water content two processes start: on the one hand the average of the line is low field shifted towards the chemical shift values of water clusters and on the other hand the number of free surface 
$-\mathrm{SiOH}$ groups is reduced, which is visible as a decline in the intensity of the $1.74 \mathrm{ppm}$ line. At water content of $17.6 \%$ nominal, which corresponds to ca. 13.7 water molecules per square $\mathrm{nm}$, the line intensity of the surface $-\mathrm{SiOH}$ groups in the CPG 10-75 is significantly reduced and in the sample with $20.9 \%$ nominal water content corresponding to $c a$. 16.4 water particles per surface unit has practically completely disappeared. This calculated $\mathrm{SiOH}$ surface densities exceed significantly the surface density characteristics of SBA-15 and MCM-41 determined by us from ${ }^{1} \mathrm{H}$ - and ${ }^{15} \mathrm{~N}-\mathrm{NMR}$ and in other silica materials, determined by thermographimetric analysis [34]. This shows that in the case of CPG, the water does not homogeneously absorb on the inner surfaces before filling the pores, as in SBA-15 and MCM-41. Instead an inhomogeneous distribution of adsorption sites, core water and outer bulk water (see below) is present. This distribution is visible in the broad signal in the spectra ranging from ca $6 \mathrm{ppm}$ to $3 \mathrm{ppm}$. In principle a measurement of the absolute density of $\mathrm{SiOH}$ groups in this material would necessitate the adsorption of pyridine, where silanol bound pyridine is distinguishable from bulk pyridine by virtue of the ${ }^{15} \mathrm{~N}$-chemical shift [33].

Upon further increase of the water content the network of hydrogen bonded - $\mathrm{OH}$ groups is shifted towards lower field, which is caused by the higher mole fraction of water molecules. The position of the ${ }^{1} \mathrm{H}-\mathrm{NMR}$ signal is gradually changed from $2.92 \mathrm{ppm}$ at $1 \%$ nominal water content to $3.22 \mathrm{ppm}$ at $20 \%$ nominal water content. This systematic low field shift of the line as a function of the water content is interpreted as a preferential layer wise growth of the water layer on the surface. The similar mechanism of water filling is found in SBA-15, while in MCM-41 one has a bimodal distribution of the thickness of the water layer on the surface, due to the coexistence of filled pores and pores where only the surface is covered with water [1]. There the different pore filling mechanisms were explained by the difference of the pore diameters of these materials. The larger pore diameters of $8.0 \mathrm{~nm}$ (in SBA-15) and of $10.3 \mathrm{~nm}$ (in CPG 10-75) give rise to a distribution of the thickness of the water layer on the inner pore surfaces, while the narrower pore diameter of MCM-41 $(3.3 \mathrm{~nm})$ promotes another mechanism of the pore filling, where completely filled pores or pore regions coexist with pores, where only the silica surface is covered with water.

The proposed filling mechanism for the CPG is sketched in Fig. 4. After the initial wetting of the surface and filling of defect sites (left fragment in Fig. 4), further filling occurs layerwise from the pore wall towards the center of the pore (middle fragment in Fig. 4) until finally complete filling of the pores is achieved (right fragment in Fig. 4). Upon further increase of the water content the single line at $4.94 \mathrm{ppm}$ is visible in the spectrum with $86 \%$ nominal water content. This value lies between the value for free water clusters $(5.5 \mathrm{ppm})$ and the value caused by the exchange of the surface $-\mathrm{OH}$ groups. Therefore this signal is attributed to the bulk water inside the pore channel, which is in fast exchange on the NMR time scale with the water phase on the surface. In 


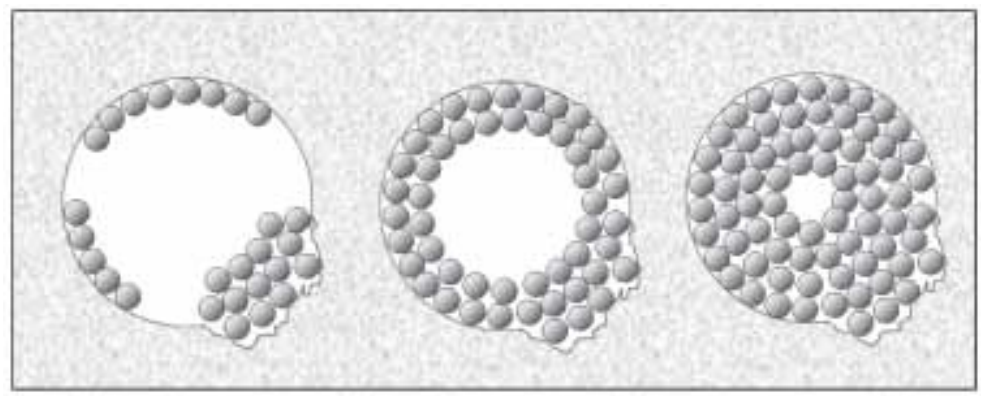

Fig. 4. Sketch of the proposed pore filling mechanism of CPG 10-75. Initially only the surface and some defect sites are covered with water; later a radial growth towards the pore axis is monitored.

the following this phase is called the core water. Upon closer inspection of the line shape, a shoulder on the low-field side at $5.3 \mathrm{ppm}$ is visible. Its origin is discussed below.

Finally in the spectrum of the sample overloaded with water (134\% nominal water content) a single asymmetric line is found, which at deconvolution gives two signals, a broad component centered at $5.0 \mathrm{ppm}$ and a narrow component at $5.3 \mathrm{ppm}$. The first broad component is attributed to the core water. The narrow line at $5.3 \mathrm{ppm}$ is attributed to water outside of the silica grains. From the pore volume of the sample, the calculated water excess is $34 \%$ nominal. Thus the integral intensity of this line should be only $34 \%$ nominal of the integral intensity of the core water. The line shape analysis of the spectrum reveals that both lines have roughly the same integral, i.e. they correspond to the same amount of water. Thus the amount of water outside the pores is a factor of two larger than expected from the pore volume.

In principle there are two different interpretations for this finding: (i) In the small pore diameter samples we always observed a complete filling of the pores before noticeable amounts of bulk water outside the pores were observed. If this would be also the case in the CPG sample the excess of outer water compared to the core water would lead to the conclusion that part of the pore volume of the CPG 10-75 is inaccessible to the water and thus not filled with water molecules. From the excess intensity one would estimate that roughly $25 \%$ nominal of the pore volume is inaccessible to the water. (ii) Owing to the larger pore width and the smaller specific pore surface the equilibrium ratio of inner to outer water is shifted in favor of the outer bulk water.

Since an inaccessible pore volume of $25 \%$ is far too high to be reasonable, we can exclude the first explanation. Thus it follows that complete pore filling is likely to be achieved only when the porous glass is totally immersed in bulk water in CPG-10-75. This will happen probably also in other high diameter silica. This conclusion explains also the presence of the low-field shoulder 
in the $86 \%$ nominal sample. Already here a substantial amount of free water is outside the pores even so the accessible pore volume is not yet completely filled.

In addition to the relatively narrow components of the core water, the surface-monolayer and the outer water, a broad component with low intensity covering the scale from $2 \mathrm{ppm}$ to $8 \mathrm{ppm}$ is observed in the spectra at intermediate water content. This broad component can be explained by several scenarios of water binding, which are not mutually exclusive, namely $(i)$ outer bulk and surface water; (ii) strongly bound immobilized water molecules, where residual dipolar interactions among the protons are too strong to be fully removed by MAS; (iii) line broadening caused by chemical exchange between protons; (iv) possible defect sites in the silica with distributions of chemical shifts, for example due to the formation of water dimers or trimers inside these defects.

Finally, in all spectra, except the nominally dry sample, one or two very weak lines between $0.8 \mathrm{ppm}$ and $1.79 \mathrm{ppm}$ are observed. The following facts help to attribute them: $(i)$ their positions are lower field shifted at increasing water content in the CPG 10-75, (ii) these lines do not appear in the nominally dry sample and finally, (iii) their chemical shifts are close to the value of monomeric water. Thus one can assume that these lines stem from the molecules of water vapor occurring inside or outside the pores.

\section{Summary and conclusion}

The desorption of water in mesoporous silica material CPG 10-75 was studied employing ${ }^{1} \mathrm{H}-\mathrm{MAS}$ solid state NMR spectroscopy. Various NMR lines of -OH hydrogen atoms are observed at different water contents in the pores. The assignment of the line positions to individual types of $-\mathrm{OH}$ hydrogen was done by virtue of their chemical shift. All observed hydrogen atoms are either surface $-\mathrm{SiOH}$ groups or hydrogen bonded water molecules. It is found that there exist some stronger bound water molecules in the CPG 10-75, which are not removable by heating at a vacuum pump. As a tentative assignment these water molecules are attributed to surface defects or inaccessible cavities in the CPG 10-75. At intermediate filling levels a principal single line with continuously varying chemical shift is observed. This leads to the conclusion that the filling of the pore grows radially from the pore surface towards the pore axis, similar to the previously studied SBA-15. This result has important consequences for catalytic applications of this material. Moreover it is shown that, owing to the large pore diameter and lower surface area, already at moderate pore filling a substantial amount of outer bulk water is formed, which is in equilibrium with the inner pore water. Finally it has been shown that water can be employed ( $i$ ) to determine the surface density of $-\mathrm{SiOH}$ groups and (ii) as a sensor for surface inhomogeneity. From these results two new questions arise, namely if there are H/D isotope effects on the filling mechanisms and what is the tem- 
perature and pore diameter dependence of the pore condensation in the silica pores. These questions are currently studied in our lab.

Finally our study shows that mainly the pore diameter is responsible for the behavior of water inside the silica pores and thus well ordered silica materials like SBA-15 are good model compounds for the study of less ordered silica, as for example the technical silica materials employed in food processing.

\section{Acknowledgement}

Financial support by the Deutsche Forschungsgemeinschaft, SFB-448, the Graduate School GK-788 and "Berliner Programm zur Förderung der Chancengleichheit für Frauen in Forschung und Lehre", is gratefully acknowledged.

\section{References}

1. B. Grünberg, T. Emmler, E. Gedat, I. Shenderovich, G. H. Findenegg, H. H. Limbach, and G. Buntkowsky, Chemistry 10 (2004) 5689.

2. A. Vyalikh, T. Emmler, E. Gedat, I. Shenderovich, G. H. Findenegg, H.-H. Limbach, and G. Buntkowsky, Solid State NMR 28 (2005) 117.

3. E. W. Hansen, M. Stöcker, and R. Schmidt, J. Phys. Chem. 100 (1996) 2195.

4. M. J. Setzer, J. Colloid Interf. Sci. 243 (2001) 193.

5. R. Kimmich and H. W. Weber, Phys. Rev. B 47 (1993) 788.

6. R. Kimmich, F. Klammler, V. D. Skirda, I. A. Serebrennikova, A. I. Maklakhov, and N. Fatkullin, Appl. Magn. Res. 4 (1993) 425.

7. R. Kimmich, NMR Tomography Diffusometry Relaxometry. Springer, Berlin (1997).

8. J. Bodurka, A. Gutsze, A., G. Buntkowsky, and H.-H. Limbach, Z. für Phys. Chem. 190 (1995) 99.

9. J. Bodurka, G. Buntkowsky, A. Gutsze, and H.-H. Limbach, Z. Naturforsch. 51c (1996) 81.

10. J. Bodurka, G. Buntkowsky, R. Olechnowicz, A. Gutsze, and H.-H. Limbach, Colloids and Surfaces A 115 (1996) 55.

11. J. Bodurka, A. Gutsze, G. Buntkowsky, and H.-H. Limbach, Z. Phys. Chem. 190 (1995) 99.

12. K. Overloop and L. van Gerven, J. Magn. Reson. Ser. A 101 (1993) 179.

13. K. Morishige and H. Iwasaki, Langmuir 19 (2003) 2808.

14. Y. Hirama, T. Takahashi, M. Nino, and T. Sato, J. Colloid Interf. Sci. 184 (1996) 349.

15. T. Ishizaki, M. Maruyama, Y. Furukawa, and J. G. Dash, J. Cryst. Growth 163 (1996) 455.

16. E. W. Hansen, E. Tangstad, E. Myrvold, and T. Myrstad, J. Phys. Chem. B 101 (1997) 10709.

17. K. Morishige and K. Nobuoka, J. Chem. Phys. 107 (1997) 6965.

18. J. M. Baker, J. C. Dore, and P. Behrens, J. Phys. Chem. B 101 (1997) 6226.

19. T. Takamuku, M. Yamagami, H. Wakita, Y. Masuda, and T. Yamaguchi, J. Phys. Chem. B 101 (1997) 5730.

20. K. Morishige and K. Kawano, J. Chem. Phys. 110 (1999) 4867.

21. C. Favire, D. Bellet, and G. Dolino, Eur. Phys. J. B 7 (1999) 19.

22. J. Dore, Chem. Phys. 258 (2000) 327. 
23. J. S. Beck, J. C. Vartuli, W. J. Roth, M. E. Leonowicz, C. T. Kresge, K. D. Schmitt, C. T.-W. Chu, D. H. Olson, E. W. Sheppard, S. B. McCullen, J. B. Higgins, and J. L. Schlenker, J. Am. Chem. Soc. 114 (1992) 10834.

24. D. Zhao, J. Feng, Q. Huo, N. Melosh, G. H. Fredrickson, B. F. Chmelka, and G. D. Stucky, Science 279 (1998) 548.

25. E. Gedat, A. Schreiber, G. Findenegg, I. Shenderovich, H.-H. Limbach, and G. Buntkowsky, Magn. Res. Chem. 39 (2001) 149.

26. E. Gedat, A. Schreiber, J. Albrecht, I. Shenderovich, G. Findenegg, H.-H. Limbach, and G. Buntkowsky, J. Phys. Chem. B 106 (2002) 1977.

27. W. Masierak, A. Gutsze, and G. Buntkowsky, Z. Phys. Chem. 217 (2003) 1613.

28. T. Emmler, S. Gieschler, H. H. Limbach, and G. Buntkowsky, J. Mol. Struct. (2004) 29.

29. S. Bhosale, G. T. Li, F. T. Li, T. Y. Wang, R. Ludwig, T. Emmler, G. Buntkowsky, and J. H. Fuhrhop, Chem. Comm. 28 (2005) 3559.

30. U. Ciesla and F. Schüth, Microporous Mesoporous Mater. 27 (1999) 131.

31. L. D. Gelb, K. E. Gubbins, R. Radhakrishnan, and M. Sliwinska-Bartkowiak, Rep. Prog. Phys. 62 (1999) 1573.

32. R. Anwander, I. Nagl, M. Widenmayer, G. Engelhardt, O. Groeger, C. Palm, and T. Röser, J. Phys. Chem. B 104 (2000) 3532.

33. I. Shenderovich, G. Buntkowsky, A. Schreiber, E. Gedat, S. Sharif, J. Albrecht, N. S. Golubev, G. H. Findenegg, and H. H. Limbach, J. Phys. Chem. B 107 (2003) 11924.

34. R. Mueller, H. K. Kammler, K. Wegner, and S. E. Pratsinis, Langmuir 19 (2003) 160.

35. M. Nakahara and C. Wakai, Chem. Lett. 21 (1992) 809. 